\title{
Welcome to the inaugural issue of Fractal Geometry and Nonlinear Analysis in Medicine and Biology (FGNAMB). We invite your participation
}

\author{
Bianciardi G* \\ Department of Medical Biotechnologies, University of Siena, Via delle Scotte 6, 53100 Siena, Italy
}

If you read these pages, you certainly know the seminal book by Benoit B. Mandelbrot, The Fractal Geometry of Nature (1982). In his Masterpiece, Benoit provides both a mathematical model and a description for many of the seemingly complex forms and patterns in nature, showing that fractal patterns are extremely familiar in our World, since nature is full of fractals. Rivers, coastlines, mountains, clouds and galaxies. And life.

Living nature has used fractal designs for some billions of years. Pinecones, sunflowers, ferns, fossilized ammonites create forms adding the same pieces, growing and twisting at a constant rate. A tree is formed by a spout branching, each of the branches branching again and again. The shape of neurons from the human cortex The fractal branching of brain cells creates the incredibly complex network that builts our mind. Our lungs are branching fractals, in order to built an enormous surface area to exchange oxygen and $\mathrm{CO}_{2}$.

The complex structure of living creatures is difficult (impossible!) to reduce to simple geometric descriptions, functional processes have nonlinear properties. We need Mandelbrot's fractal geometry and nonlinear algorythms to describe life.

The concept of fractal dimension is attractive. The possibility for a geometric, repetitive structure to have a dimension different from the topological dimension enable to work about the underlying function. In the brochial tree, a substance to be moved (air, a 3D object) travels between the exchange surfaces $(D=2)$ via a multi pipe tree $(D=1)$, gaining better efficiency in transporting the $3 \mathrm{D}$ substance if the real (fractal) dimension approaches the vaue 3 . The superficial layers of the cerebral convolutions possess a geometry with fractal dimension near to 3, while its surface has topological dimension equal to 2: the convolution pattern is so efficient to be nearly "space-filling", in order to reach as many spatial coordinates as a solid 3D structure. The fractal dimension permits TO MEASURE this common characteristic that share shape and function.

Since the pioneering work of Edward Lorenz "Nonperiodic deterministic flow in......" an increasing number of studies has been concerned with deterministic nonlinear systems whose signals are aperiodic and erratic, just if they had emerged from a stochastic process. These systems may develop an exponential divergence after minute changes of initial conditions: they are defined as "deterministic chaos". As Lorenz taught, when plotted in a state space of variables, chaotic systems converge to domains with a fractal dimension: deterministic chaos is closely related to fractal geometry. Deterministic chaos and fractal properties have been demonstrated within the real world, e.g. biology and pathology.

Look at the heartbeat. The heart is a deep integrator of what happens inside the body. The main cardiac physiological pacemaker, the sinus node, sums inputs from the respiratory system and integrates stochastic inputs from the autonomous nervous system. The interbeat intervals of the heart, known as R-R, result from a complex superposition of multiple physiological processes at their respective characteristic time scales, so resulting with multifractal properties. Perhaps the first Authors that point on fractal characteristics of the heartbeat are Goldberger \& West. Few years and wavelet-based analysis of the heartbeat revealed its ability to distinguish among healthy and disease, prediction of sudden cardiac death was performed by fractal analysis of the heartbeat intervals, or analyzing the heart rate variability as a stochastic process, decomposing the series in deterministic terms and in noise ones, was demonstrated the ability to quantify the state of the autonomous nervous system, as well of blood pressure control. A lot of papers arose proposing the effectiveness of nonlinear parameters like Detrended Fluctuation Analysis, Approximate Entropy indices, and various chaotic parameters to assist in diagnostic and prognostic evaluations. In effect, even if, probably, heartbeat series are not really chaotic, being the heartbeat a nonstationary, multifractal, process with strong nonlinearity, it is widely accepted that we can use chaotic indices as a tool to analyze the nonlinear properties of the heartbeat intervals, in order to support differential diagnosis.

Well, it's the time to show the powerful of fractal/nonlinear approaches in pathology and clinical medicine, ROC curves and double blind experiments are welcome, here! (and without deny the importance to underline the usefulness of fractal/nonlinear approaches in other fields like biology or biophysics). FGNAMB would be a working board, able to provide input concerning general trends in these

Correspondence to: Bianciardi G, Department of Medical Biotechnologies, University of Siena, Via delle Scotte 6, 53100 Siena, Italy, E-mail: giorgio.bianciardi@unisi.it

Received: March 18, 2015; Accepted: March 20, 2015; Published: March 22, 2015 
fascinating fields. The goal of FGNAMB journal is to provide a forum for the demonstration of the importance of fractals and nonlinear analysis in medicine, biology and biophysics, in order to continuously explore these worlds in mathematics, science and technology.

We invite your participation. Please send all manuscript submissions (Editorial, Letter to the Editor, Review, Research Article, Technology, Case Studies: http://oatext.com/ArticleTypes-Format. php), according the manuscript preparation: http://oatext.com/ Manuscript-Preparation.php to the Editor-in-Chief: editor.fgnamb@ oatext.com, giorgio.bianciardi@unisi.it

Copyright: $@ 2015$ Bianciardi G. This is an open-access article distributed under the terms of the Creative Commons Attribution License, which permits unrestricted use, distribution, and reproduction in any medium, provided the original author and source are credited. 